\title{
Gradhiva
}

GRADHIV

Revue d'anthropologie et d'histoire des arts

$4 \mid 2006$

Le commerce des cultures

\section{Le musée d'un lieu saint en chantier}

La Sagrada Família entre archives et achèvement virtuel

The museum of a holy place under construction. The Sagrada Familia between archives and virtual completion

\section{Mathieu Claveyrolas}

\section{OpenEdition \\ Journals}

\section{Édition électronique}

URL : http://journals.openedition.org/gradhiva/590

DOI : 10.4000/gradhiva.590

ISSN : 1760-849X

\section{Éditeur}

Musée du quai Branly Jacques Chirac

\section{Édition imprimée}

Date de publication : 1 novembre 2006

Pagination : 71-83

ISBN : 2-915133-44-1

ISSN : 0764-8928

\section{Référence électronique}

Mathieu Claveyrolas, «Le musée d'un lieu saint en chantier », Gradhiva [En ligne], 4 | 2006, mis en ligne le 15 novembre 2009, consulté le 19 avril 2019. URL : http://journals.openedition.org/gradhiva/590 ; DOI : 10.4000/gradhiva.590

Ce document a été généré automatiquement le 19 avril 2019.

(C) musée du quai Branly 


\title{
Le musée d'un lieu saint en chantier
}

\author{
La Sagrada Família entre archives et achèvement virtuel* \\ The museum of a holy place under construction. The Sagrada Familia between \\ archives and virtual completion
}

\section{Mathieu Claveyrolas}

1 Le 19 mars 1882, jour de la Saint-Joseph, le libraire Josep M. Bocabella, président de l'Associació Espiritual de Devots de Sant Josep, pose à Barcelone la première pierre du Temple Expiatori de la Sagrada Família (temple expiatoire de la Sainte Famille), église catholique dont il patronne la construction. Cent vingt-quatre ans ont passé et deux constats s'imposent.

2 D'un côté, seules la crypte et deux façades surmontées de quatre clochers chacune sont terminées, mais les nefs et certaines parties du cloître, entre autres espaces, sont bien avancées. L'œuvre est donc encore en plein chantier; l'architecte en chef Jordi Bonet parle d'un peu plus de $50 \%$ des travaux réalisés, et pense que les « enfants des enfants de nos enfants » la verront achevée. En attendant, peut-on lire dans la revue Templo ${ }^{1}$, fondée par les promoteurs du projet, «Barcelone ne sera pas vraiment Barcelone tant que le Grand Temple de la Famille de Nazareth et des Familles Chrétiennes ne sera pas une réalité vivante, lieu de prière et d'eucharistie » (Templo 1961 : 16).

D'un autre côté, l'état d'inachèvement n'empêche pas les huit clochers terminés par des pinacles polychromes à 100 mètres de hauteur d'être d'ores et déjà le symbole le plus fort de Barcelone et de la Catalogne dans le monde entier, la première attraction touristique de la ville et l'une des œuvres d'art les plus originales au monde.

4 Le projet de construction de la Sagrada Família, au départ pensé comme une réplique de l'église italienne de Loreto, ne prit une ampleur hors du commun qu'avec l'architecte Antoni Gaudí, qui succéda à Paula del Villar en 1883 à la tête du chantier. Gaudí avait déjà travaillé à la rénovation d'autres bâtiments religieux, mais il est surtout connu pour ses constructions de parcs, fontaines et maisons qui donnent son empreinte au paysage urbain de Barcelone. Considérant la Sagrada Família comme son "grand œuvre », il 
refusa toute commande à partir de 1918 pour s'y consacrer exclusivement, jusqu'à sa mort en 1926.

5 Gaudí s'attacha à rendre le plus compréhensibles possible les enseignements du catholicisme en faisant de son œuvre une "Bible en pierre ", foisonnante de symboles catholiques. Toute la mythologie chrétienne sera illustrée : 12 clochers pour les apôtres, 4 autres pour les évangélistes, 1 pour la Vierge et le plus grand pour Jésus. Quant aux scènes représentées sur les façades, de celle de la Nativité (face au soleil levant) à celle de la Résurrection en passant par celle de la Passion (face au soleil couchant), elles ont pour mission d'illustrer d'une manière claire et vivante les mystères du Christ. Mais l'imagination gaudienne, ses références mystiques et esthétiques originales et audacieuses brouillent souvent le message de son œuvre, souvent interprétée par le visiteur comme un " délire artistique et spirituel », une "bande dessinée religieuse », en tout cas une église qui « change des autres églises ». Auteur d'un ouvrage sur l'architecte, Juan José Navarro Arisa (2002) se demande à son tour: "Qui fut Antoni Gaudí? Un créateur illuminé de visions psychédéliques ou un homme de foi absolue ${ }^{2}$ ?»

6 La Sagrada Família, construite au départ aux marges de la ville, est aujourd'hui située dans le quartier essentiellement résidentiel de l'Eixample (littéralement «l'Expansion »). Le terrain sur lequel se bâtit l'église, qui est la propriété de l'association fondatrice de Bocabella, occupe un bloc délimité par quatre rues perpendiculaires deux à deux. La Sagrada Família est ainsi localisée à plusieurs kilomètres du centre historique de Barcelone. Celui-ci, appelé Barri gòtic (" quartier gothique »), a pour centre la cathédrale médiévale de la ville, symbole du «vrai » gothique face au néogothique de la Sagrada Família.

7 L'église, de par la monumentalité de son projet, la personnalité de son architecte et l'importance de son identité propre, usurpa rapidement le nom de « cathédrale ». Elle fait office de paroisse : la crypte, seul espace sanctifié, sert de lieu de culte intérimaire en abritant chaque jour (depuis plus d'un siècle) plusieurs messes, dans l'attente de l'achèvement et de la sanctification de l'ensemble du bâtiment.

Une des originalités du chantier de la Sagrada Família tient dans le fait d'intégrer dans ses murs un musée dont la vocation est de présenter le chantier au visiteur. Le musée a été inauguré en juin 1961 et ne cesse de s'étendre depuis quelques années, notamment après l'agrandissement réalisé en 1992, année des Jeux olympiques à Barcelone. Officiellement appelé Museu del Temple, il est présenté dans les brochures comme «le musée le plus visité de Barcelone». Il faut pourtant rendre justice aux autres nombreux musées barcelonais : ce ne sont pas les collections ni même les pièces particulières du musée du temple, si intéressantes soient-elles, qui en font l'originalité et l'attrait mais bien, d'une part, sa situation au cœur de la Sagrada Família et, d'autre part, le concept muséographique en tant que tel. Ce concept consiste à faire d'un chantier en construction un patrimoine vivant, à la fois par son statut actuel et par son évolution permanente.

9 L'ambition de cet article est, à travers l'ethnographie du musée, d'engager une réflexion d'ensemble sur le chantier de la Sagrada Família, cet immense terrain permettant l'étude anthropologique du religieux en train de se faire. En effet, ce que la Sagrada Família donne à voir, c'est la construction concrète et au présent d'un lieu sacré. Cette situation unique devrait permettre d'étudier les carrefours entre anthropologie religieuse et histoire de l'art, tourisme et dévotion, ou encore entre universel catholique et régionalisme catalan. Il serait alors possible de travailler à une approche anthropologique de la présence divine, 
aussi bien inhérente au lieu saint sanctifié que construite au quotidien par les acteurs locaux (ouvriers-artistes, dévots, officiants, visiteurs, etc.).

J'étudie ici les modalités et les enjeux de l'articulation originale d'une église et d'un musée, tels que l'espace et la muséographie la mettent en scène. Je montrerai comment l'ambition muséale cristallise cette ambiguïté liée aux différents statuts concomitants de la Sagrada Família en tant que chantier, lieu saint et œuvre d'art.

11 Il s'agit également de rendre compte de l'importance stratégique (pour la mise en valeur du chantier) de la temporalité dans ce musée qui s'intéresse à la fois au passé, au présent et au futur de l'œuvre qu'il expose : comment un musée peut-il inscrire dans le temps cette « Bible en pierre » qui, en tant que chef-d'œuvre et en tant que lieu saint, semblait doublement vouée à l'atemporalité ?

\section{De l'église au musée}

12 Comparer le musée à un lieu saint n'est pas une nouveauté ${ }^{4}$. Il serait étrange que le musée d'une église déroge à la règle. De fait, un pictogramme réclame le silence à l'intérieur du musée, comme on le réclame du visiteur d'un lieu saint ; on évoquera comme autre point commun du musée et du lieu saint l'admiration-dévotion respectueuse et l'esprit d'éloge unanime assez coutumiers aux musées consacrés à des œuvres d'art, et plus encore aux « chefs-d'œuvre ». Mais ici, la sanctuarisation du musée est plus poussée encore. Il n'y a pas seulement, de l'église au musée, continuité dans l'espace, mais également inclusion. On pénètre en effet dans le musée en descendant une rampe menant dans la crypte qui l'abrite sous ses arches en croisées d'ogives.

13 Le droit d'entrée couplant obligatoirement les deux visites (chantier de l'église et musée) est de 8 euros. Une fois le guichet franchi, nul ticket, paiement, ni contrôle supplémentaire ne viennent rompre la fluidité entre l'église à venir et le musée. De fait, la plupart des visiteurs prolongent leur visite de l'église par celle du musée. C'est d'ailleurs ce à quoi les incitent les guides des groupes de touristes et les audioguides individuels.

Remarquons d'abord que l'utilisation du musée comme espace de propagande de l'œuvre et donc d'incitation aux donations existait dès l'ouverture du musée (1961). Ainsi que le remarque la revue des dévots de saint Joseph dans l'ultime paragraphe de son compte rendu de l'inauguration : «[...] en quittant l'exposition, un petit panneau, à peine visible [sic], nous rappelle le devoir expiatoire que nous avons tous contracté pour la construction du Temple.» (Templo 1961: 16) Aujourd'hui, il est précisé à plusieurs reprises (texte des audioguides, brochures, etc.) que «les entrées touristiques sont considérées comme des dons ». La visite du chantier est par ailleurs balisée de nombreuses boîtes à donations, certaines modernes prévoyant le paiement par carte, d'autres plus traditionnelles, en pierre, de forme grossièrement cubique avec, gravée en lettres gothiques, la mention "aumônes ». C'est l'un de ces troncs en pierre que l'on trouve dans le musée, situé devant le panneau résumant la chronologie du chantier. Situation idéale puisque, si l'inscription gravée « une grâce de charité » rappelle la vertu religieuse du geste, l'aumône-donation fait explicitement appel au rôle de chacun dans le financement des travaux. On trouve la légende suivante, non pas gravée cette fois, mais inscrite en huit langues sur un cartel fixé au tronc : « Les donations rythment les travaux. Nous ne recevons pas d'argent des institutions publiques. Nous vous remercions de votre collaboration. » L'audioguide du musée explique qu'il s'agit là d'une prescription du 
fondateur Bocabella, qui exigea que l'œuvre entière, en tant que temple expiatoire, soit exclusivement construite à partir de dons individuels ${ }^{5}$. Le projet de construction de la Sagrada Família visait en effet à expier, en construisant une "cathédrale des pauvres ", « les péchés de l'égoïsme bourgeois et de l'extrémisme athée » (Bonet : 4). Il s'agissait de lutter contre la perte de vitesse du christianisme en ces années de révolution industrielle, ce à quoi « la Providence veillera puisque à la Sagrada Família, expliquait Gaudí, tout est providentiel ${ }^{6} »$.

Notons également que les nombreuses représentations de l'œuvre finie, y compris dans le musée, illustrent clairement la volonté gaudienne d'une église ouverte sur l'espace extérieur. Les parcs entourant le Temple, appelés « parc de Gaudí » et « parc de la Sagrada Família », ont été en partie conçus par l'architecte, qui souhaitait y planter des végétaux religieusement symboliques renvoyant à ceux sculptés sur chacune des façades (Templo 1979). Les routes, même, devaient passer sous des rampes reliant l'église aux parcs environnants. Le projet final de la Sagrada Família lui rendra son statut traditionnel : il n'y aura plus de guichet d'entrée; la visite ne sera plus payante; ce ne sera plus une "visite ». Certains touristes-fidèles hésitent encore sur le statut de ce bâtiment en construction : «Sur le chantier, on ne peut pas ne pas ressentir quelque chose de spécial. C'est un lieu spécial. Un lieu saint. » Pourtant, la rupture entre le lieu et l'extérieur vise actuellement les touristes, et seulement eux : on ne paie que pour la visite touristique (et le billet peut même être couplé, moyennant réduction, avec d'autres visites purement touristiques à Barcelone, comme celle de la maison Gaudí). La partie inférieure de la crypte consacrée aux célébrations du culte est, elle, accessible directement et gratuitement par la rue, en tout cas aux heures d'ouverture (assez restreintes: deux à trois heures par jour, à comparer aux huit à dix heures du chantier).

S'il y a bien inclusion du musée dans le chantier de l'église, le seul espace sanctifié semble lui, au contraire, conservé à l'écart du circuit touristique. Pourtant, l'inauguration du musée de la Sagrada Família s'est faite, en 1961, avec la bénédiction solennelle de l'archevêque de Barcelone (Templo 1961 : 8). De fait, là où le musée de la Sagrada Família s'avère un lieu clé, c'est qu'il fait le lien entre le chantier et la crypte, entre l'œuvre d'art et le lieu saint.

\section{« Tout musée est un tombeau »}

17 Le musée incarne concrètement ce lien : un des points forts de la visite est, tout au fond d'une des branches latérales, la vision en plongée de la tombe d'Antoni Gaudí (salle n ${ }^{\circ} 15$ ). L'obscurité, la distance (plusieurs mètres plus bas) et l'angle restreint rendent en fait la vision très difficile, voire impossible, même si la crypte, le plus souvent fermée, est toujours illuminée par deux lustres surplombant la tombe. Les audioguides proposent une halte devant cette tombe, et les guides s'arrêtent là pour commenter spécialement cette vision. L'insistance de la mise en scène sur cette séquence, qui dévoile moins qu'elle ne suscite le désir de voir, consiste effectivement à entretenir le mystère et à sanctuariser le musée, à en faire un lieu dont la vocation dépasse la seule exhibition (Galinier et Molinié 1998 : 96).

18 La personnalité de Gaudí et sa mort patrimonialisent de fait la Sagrada Família. Gaudí est entièrement aux mains de la légende, qui lui prête une véritable vocation de bâtisseur de cathédrale. La légende veut ainsi qu'il ait été tué, renversé par un tramway en l'une des rares occasions où il quitta son chantier et traversa la rue, la tête toute à ses pensées 
géniales (trop absorbé pour regarder avant de traverser la rue, en quelque sorte) et portant comme seul objet un exemplaire de la Bible... La Sagrada Família est tellement identifiée à Gaudí que les commentaires oublient souvent qu'il n'est ni l'initiateur du projet (c'est le libraire Bocabella qui a lancé le chantier et l'a confié à l'architecte Villar), ni le réalisateur de la plupart des parties visibles du bâtiment actuel, construites après sa mort (voir la chronologie en note 12).

Il était logique que Gaudí repose dans les murs de son œuvre maîtresse, dans la crypte de cette église à laquelle il consacra exclusivement les huit dernières années de sa vie au point d'y installer son atelier et d'y vivre quasiment en reclus. L'atelier de Gaudí est abondamment muséographié, à la fois dans une scénographie grandeur nature (dans une des salles des écoles, servant d'annexe au musée) et par l'exposition de photographies d'époque montrant une cellule spartiate avec lit et table de travail. Les photographies commentées amorcent la sanctification de Gaudí, insistant sur l'état de l'atelier « comme il était le jour de sa mort », et sur cette table sur laquelle il «travaillait et mangeait ${ }^{8}$ " avec, «enroulé dans un chiffon, le repas qu'on lui avait préparé, fait de deux petites tranches de pain au miel et d'une grappe de raisin ».

L'audioguide réserve à ce "studio-atelier » une des huit séquences qu'il consacre au musée (sur une vingtaine pour l'ensemble de la visite de la Sagrada Família). Un lecteur y imite la voix supposée chevrotante de Gaudí vieillissant disant : «Mes grands amis sont morts. Je n'ai plus ni famille, ni client, ni fortune, ni rien. Alors je peux me consacrer uniquement au Temple.»

21 Le fantôme de Gaudí est omniprésent dans le musée : outre ses œuvres, des photographies le représentent, et l'on trouve même, exposé devant la fenêtre donnant sur sa tombe, un buste de l'architecte réalisé, précisent le cartel et les commentaires de l'audioguide, à partir de son masque mortuaire ${ }^{9}$. Ajoutons la démultiplication de la représentation de Gaudí mort. Il est présent sous la forme de cette tombe à peine visible, mais aussi sous celle de l'image de la tombe filmée en direct et figée sur un téléviseur, du masque funéraire, de deux autres panneaux présentant cinq photographies de toutes les étapes de son enterrement (jusqu'à l'inhumation dans le Temple) et d'un dessin de Gaudí sur son lit de mort...

22 L'incorporation de Gaudí dans l'église et l'assimilation de l'œuvre à son initiateur ont, du point de vue muséographique, une conséquence majeure. Le « musée du Temple » devient souvent, y compris sur les panneaux indiquant son entrée, le «musée de Gaudí » (le pictogramme étant un portrait de l'architecte). La revue Temple écrit au moment de l'agrandissement du musée en 1992 : «Ce musée est surtout dédié à la figure de Gaudí à travers sa relation au Temple. » (Temple 1992 : 18) Gaudí vivait et créait dans l'œuvre qui abrite aujourd'hui le musée. Ce musée du Temple expose donc des objets fabriqués dans ce même Temple. Ainsi, qu'il évoque le culte de Gaudí ou, plus indirectement, l'une ou l'autre de ses créations, jamais l'objet muséographié ne connaît ce changement de destination, de la production à l'exposition, qui définit habituellement la pièce de musée. On notera que le maître et sa légende font école. Le sculpteur Subirachs vit et crée à son tour au cœur même de la Sagrada Família. Il explique qu'ainsi, lorsqu'il a des doutes, il lui suffit de se promener dans l'espace du chantier pour trouver la solution, la réponse ( $L a$ Sagrada Familia, DVD), comme si l'œuvre parlait d'elle-même, comme s'il ne s'agissait que de réaliser, de concrétiser une œuvre déjà définitivement créée et achevée par Gaudí. en procès officiel de béatification, et les marques de dévotion affluent autour de sa tombe, 
ce lieu symboliquement à l'intersection de l'espace consacré et de l'espace touristique, et à l'intersection de l'église et du musée. Les responsables actuels n'ont-ils pas entrepris d'ouvrir un nouvel accès à la crypte à partir du musée afin de répondre à l'intérêt croissant pour la tombe de Gaudí de la part de visiteurs-dévots qui y allument des bougies et y déposent des fleurs? En 2000 déjà, une journaliste notait que «la tombe de l'architecte Antoni Gaudí commence à devenir un centre de pèlerinage » (López 2000). Non seulement touristes et fidèles sont invités à faire des dons, dans un tronc jouxtant la tombe, afin de couvrir les nombreux frais engagés par l'association "pro-béatification ", mais des livrets les appellent à participer à l'avancement de ce processus en témoignant des miracles dont ils ont été les bénéficiaires suite à des actes de dévotion au maître architecte.

Ce projet de béatification de l'architecte s'enracine dans la volonté de ressusciter son souvenir à travers la commémoration de sa « vision » de l'œuvre. La présence du tombeau de Gaudí contredit ici radicalement la sentence de Segalen. Loin de «cette pétrification dont tant d'institutions muséales de simple conservation ont été les victimes » (Michelet 2001 : 21), la scénographie autour du tombeau sert ici la vocation du musée, qui est d'affirmer la continuité entre chantier (espaces à venir qui abriteront à terme le culte) et lieu consacré (crypte où se célèbrent, pour l'heure, les offices). Il s'agit également avec cette scénographie du tombeau de rendre la vie à Gaudí : « Son esprit vit encore. » (Templo 1961 : 16) La muséographie participe à la sanctuarisation d'une église en chantier et à la sanctification en cours de son architecte. Nul doute que pareille mise en lumière de la dimension exceptionnelle de l'architecte facilite à son tour un processus de patrimonialisation de l'œuvre. L'Unesco, qui avait inscrit sur la liste du Patrimoine mondial de l'humanité trois ouvrages barcelonais de Gaudí en 1984, y a récemment ajouté la Sagrada Família, eu égard à sa " contribution créative exceptionnelle et remarquable au développement de l'architecture et de la construction de la fin du XIX siècle et du début du xxe siècle $^{10} »$.

Le musée, dont la fonction au sein d'une église à mi-chantier semblait peu évidente, assoit définitivement sa légitimité en tant que lieu de conservation, de mémoire et d'hagiographie d'un patrimoine unique, mais aussi en tant que lieu de construction de la légende.

\section{Contextualiser le chantier en récapitulant la visite}

Le musée de la Sagrada Família est un anti-musée au sens où il n'expose que des objets non sortis de leur contexte de production. Pourtant, que le musée soit si peu décalé par rapport à l'œuvre muséographiée, à tel point intégré, fusionnel, redondant dans sa vocation de présentation d'une œuvre au cœur même de l'œuvre en question, ne l'empêche pas de proposer une contextualisation qui donne son intérêt et sa profondeur à l'objet muséographié.

Il s'agit d'abord de resituer le chantier présent dans le temps. Le texte qui introduit l'audioguide est à cet égard très explicite, présentant le visiteur comme le " témoin d'un monument insolite, un Temple toujours en construction, une œuvre vive, contemporaine qui continue de prendre forme jour après jour, un Temple dans lequel se rencontrent à la fois le passé, le présent et le futur.» 
28 La scénographie du musée est, dans ses grandes lignes, chronologique. La salle $n^{\circ} 1^{11}$ récapitule justement la chronologie du chantier, les dates importantes ${ }^{12}$; les salles 2 à 7 s'intéressent aux différents ouvrages de Gaudí, souvent présentés comme des ébauches de son grand œuvre; les salles 8 à 16 sont dominées par les maquettes du Temple, présentant les espaces réalisés ou à venir ; la salle 17 est quasi uniquement consacrée aux photographies, qui retracent notamment les différentes phases du chantier ; la dernière salle, $\mathrm{n}^{\circ} 18$, présente les plans et les dessins de l'œuvre achevée.

Le visiteur trouve dès l'entrée une présentation claire du chantier, de ses étapes passées et de ce à quoi l'œuvre achevée ressemblera. Comme la revue Templo le prévoyait à l'inauguration, le musée permet « aux visiteurs de se faire, en très peu de temps, une idée précise de ce que furent quatre-vingt-quinze années d'histoire du Temple» (Templo 1961 : 9).

Mais ce parcours est souvent emprunté, y compris par les guides, en sens inverse : les deux ouvertures, par chacune des façades de l'église, sont fléchées « entrée » (du musée). Le parcours à rebours du temps (on commence par le spectacle des projets terminaux avant de passer aux étapes intermédiaires puis à la fondation du chantier) est tout aussi satisfaisant. Celui qui entre dans le musée vient de visiter, d'une façade à l'autre par l'intérieur des nefs, et de bas en haut en montant par l'intérieur des clochers, un espace pluriel dans lequel il a du mal à se situer. Les questions les plus fréquentes sont : «Qu'estce qui est fini ? Que reste-t-il à construire ? ", trahissant une désorientation et un certain tournis devant l'ampleur du monument dont l'état de chantier vient encore brouiller la perception. C'est en discutant de ces questions entre eux que les visiteurs pénètrent dans le musée. Entrant par la salle $n^{\circ} 18$, le visiteur se trouve alors face à plusieurs posters de 2 mètres de haut représentant, en coupe ou en vue de face, des dessins à échelle 1/100 du projet final de l'église. Les visiteurs remarquent rarement le cartel minimal, uniquement en catalan ("Façana Passió - carrer Sardenya E.1: $100 »$ " par exemple). Pas plus d'ailleurs qu'ils ne lisent la légende inscrite sur le poster lui-même, indiquant en écriture gothique la date (entre 1965 et 1982) et l'auteur (Ramón Berenguer) du dessin. En revanche, ils sont nombreux à s'approcher pour pointer du doigt, et souvent toucher, différentes parties du dessin. Un homme récapitule ainsi, pour lui-même et pour sa femme, la visite du chantier : « Nous, on est montés là. Et on voyait les gens qui étaient en face [à l'étage du clocher d'une façade opposée]. On leur faisait coucou, on était à 75 mètres ", dit-il après avoir consulté l'annotation sur le dessin. Le même enchaîne sur la projection par rapport à l'œuvre finie : «Et ça, ce sera l'entrée. Tu verras ça. À quelle altitude on sera ? 170 mètres! Tu as vu l'escalier qui monte jusqu'en haut qu'ils ont prévu ? Faut être en forme. Putain, c'est quelque chose. Et ils construisent un phare!» Quelques mètres plus loin, un autre visiteur, tenant pourtant à la main un guide de la Sagrada Família, regarde le cartel commun aux photographies représentant les différentes étapes du chantier et réalise : « Donc, elle a déjà plus d'un siècle!»

On a confirmation de la vocation d'outil didactique du musée dans le livre destiné au grand public que l'actuel architecte en chef de la Sagrada Família consacre au Temple. Dans ce livre (non daté) que l'on retrouve dans toutes les boutiques alentour, Jordi Bonet utilise systématiquement les allers-retours entre les photographies du chantier actuel et celles des maquettes, plans et dessins du musée afin de mieux contextualiser sa présentation ${ }^{13}$. Loin de la «déstabilisation des visiteurs" visée par certains musées d'avant-garde (Hemmet 2001 : 92), l'ambition est ici de mettre de l'ordre dans la vision de l'œuvre qu'ont les visiteurs, de les guider dans leur quête de repères, leur permettant de 
ressortir de ce chantier avec une image autre que celle, impure et confuse, d'une œuvre inachevée peuplée de grues et d'échafaudages.

\section{L'atelier de plâtre : le spectacle du temps} visite de la Sagrada Família : assister à la construction au présent du lieu saint - chefd'œuvre. Notons que la page d'ouverture du site Internet officiel de la Sagrada Família dévoile à son tour, petit à petit, de bas en haut, les clochers de l'église, comme si l'on assistait à leur construction. Il s'agit, d'autre part, de resituer l'état présent d'avancement dans les différentes temporalités du chantier. Ainsi, au sein de ce musée d'archives et d'histoire de l'art, le visiteur est rappelé à la réalité présente du chantier. Mais le temps, à la Sagrada Família, est une entité plus complexe encore, dont la séquence «atelier de plâtre » parvient à rendre compte. Car que font les artisans maquettistes? La réponse est apportée par un long cartel, un des rares du musée. Expliquant précisément de quel travail il s'agit, ce cartel donne sens au spectacle :

« Dans cet atelier, on réalise les modèles de plâtre nécessaires à la construction du Temple. Les modèles détruits durant la guerre civile y sont aussi restaurés.

Gaudí disposait d'un atelier semblable situé à peu de distance d'ici, où les modèles des différentes parties du projet étaient élaborés à une échelle $1 / 1,1 / 10$ et $1 / 25$. 
Plutôt que de travailler avec des dessins, Gaudí préférait les modèles de plâtre ; ainsi, il pouvait vérifier dans l'espace le résultat de la nouvelle architecture qu'il projetait de réaliser.

Une partie des modèles restaurés que l'on peut contempler dans ce musée sont à la base de la construction du Temple. »

Le premier paragraphe du cartel expose le rôle charnière des maquettistes dans l'articulation des différentes époques du chantier. Leur travail présent permet bien sûr de visualiser le projet à venir. Mais il est aussi de l'ordre du flash-back: la projection de l'œuvre finie passe par un retour dans le passé pour restaurer les maquettes originelles. Il ne s'agit même pas à proprement parler d'une restauration. Le travail consiste à réidentifier et re-constituer les maquettes détruites pendant l'incendie de la crypte du Temple par les révolutionnaires en 1936. L'incendie se propagea aux ateliers et détruisit l'essentiel des maquettes, dont une brigade municipale parvint à recueillir des fragments, conservés jusqu'à la fin de la guerre dans un entrepôt. Il y a eu rupture : la plupart des pièces sur l'inventaire du musée ne sont datées que d'« avant 1936 » ou d'« après 1936 ». Le successeur de Gaudí, l'architecte Domenech Sugraner, « estima que la disparition de tous les plans rendait impossible la poursuite des travaux ", explique l'audioguide, qui impute même la mort de ce dernier au découragement consécutif à cette destruction. Le chantier resta effectivement entre parenthèses quelques années, marquant le début d'un débat non encore clos sur l'opportunité de le poursuivre, en lien avec la possibilité de le faire sans trahir le projet originel ${ }^{14}$. Dans ces conditions, identifier et reconstituer les modèles en plâtre, ce travail auquel le visiteur assiste à travers la vitre, revient à rendre le projet gaudien à nouveau viable, à le ressusciter.

Le deuxième paragraphe du cartel inscrit les maquettistes dans le prolongement $d u$ maître, impression confirmée par deux photographies illustrant le cartel : l'une, en haut à droite, présente en noir et blanc "L'atelier de plâtre où Gaudí élaborait ses modèles (approx. 1922) », l'autre, en bas à droite, montre en couleurs l'atelier actuel en 1996. Ce cartel occulte ainsi l'importance de l'innovation, notamment l'assistance de l'informatique, qui permet de virtualiser les constructions à venir plus efficacement encore, technique abondamment mise à contribution par les maquettistes (voir notamment Gómez éd. 1996 : 67-76).

Le dernier paragraphe du cartel dépasse la fluidité déjà soulignée entre musée et chantier jusqu'à transformer le musée d'archives en un lieu de production de l'œuvre.

39 À travers cette mise en spectacle du travail des maquettistes à laquelle sont conviés les visiteurs, le musée joue le jeu de la reconstruction historique du chantier. Il tient ainsi la gageure de contextualiser l'œuvre, de ne pas la mettre hors temps. De fait, les photographies du musée ne cèdent jamais aux tentations de purification esthétique qui consisteraient à cacher ces grues et ces échafaudages brouillant, sur le chantier, la perception du visiteur. Même les dessins projetant l'œuvre achevée incluent les routes et les voitures. C'est un chantier vivant, un chef-d'œuvre en mouvement que promeut le musée.

\section{Mise en abyme et œuvre achevée}

40 En résumé, cette scénographie autour de l'atelier synthétise la stratégie du musée, qui est de projeter l'œuvre achevée. La visite du chantier n'étant pas toujours suffisamment édifiante, on a déjà vu comment le musée servait de support à une récapitulation de la

Gradhiva, 4 | 2009 
visite et de l'œuvre, et comment les maquettes permettaient de représenter l'œuvre achevée.

Mais le meilleur outil de projection de l'avenir, surtout quand, comme ici, il est lointain et indécis, c'est le virtuel et sa représentation. Les posters et cartes postales de la Sagrada Família, omniprésents à Barcelone, usent largement de ce procédé, représentant des photographies de l'œuvre où les échafaudages sont remplacés par la représentation des espaces tels qu'ils seront une fois achevés.

Notons que cette fonction de projection de l'œuvre finie que remplit le musée répond autant aux attentes des touristes qu'à celles des fidèles, comme l'exprime la Revue des dévots de Sant José lorsqu'elle rend compte de l'ouverture du musée en 1961: "Nous passons au grand espace central qui nous réserve l'enseignement principal de l'exposition, à la grande satisfaction de tous. Il s'agit du modèle, une maquette en plâtre à échelle $1 / 10$, de l'intérieur du futur Temple avec ses lignes de colonnes et la vision quasi réelle de ce que seront les cinq nefs intérieures de notre cathédrale. Une figure humaine, reproduite à la même échelle, nous donne une idée de l'immensité et de la grandeur de ces nefs. " (Templo 1961: 10-16) Anticipant sur l'église achevée, le musée répond dès aujourd'hui à ce besoin de voir pour croire contemporain de l'art des cathédrales médiévales, ainsi que le souligne Roland Recht (1999). Le passage quasi obligé sous cette maquette des nefs (salle ${ }^{\circ} 8$ ) guide le visiteur vers l'expérience d'un espace vertical " modèle réduit » inscrit dans la crypte d'une église en construction.

On trouvera sur le site Internet de la Sagrada Família une visite virtuelle de l'église proposant au visiteur de cliquer sur différents espaces de l'œuvre, commentés et dans lesquels il peut se déplacer en trois dimensions. Mais le site présente également le musée... et propose, à nouveau, sa visite virtuelle. L'on peut notamment cliquer et obtenir, là encore, la vision en trois dimensions de l'atelier de plâtre. La mise en abyme est alors totale: visiter sur Internet la scénographie de la construction d'une œuvre visant la mise en scène du divin ${ }^{15} \ldots$

Si l'on rappelle l'inclusion du musée dans l'église, et la tombe de Gaudí dans l'église et dans le musée, il devient frappant que le procédé de mise en abyme des objets constitue l'une des caractéristiques principales de la scénographie du musée. On peut étudier ce procédé muséographique à travers une séquence spécifique. Elle est située salle $\mathrm{n}^{\circ} 16$, en face de l'atelier de plâtre, et expose différentes représentations des futures fenêtres de la nef centrale :

1. À gauche, deux dessins de Berenguer: l'un montre les fenêtres en coupe, l'autre en vue de l'intérieur. Ils sont réunis sous un même cadre de 2 mètres de haut environ (avec titre, échelle, auteur et date).

2. Au centre, la maquette originale en plâtre, à échelle $1 / 10$, des mêmes fenêtres: un personnage aide à nouveau à visualiser l'échelle. La maquette est l'œuvre de Gaudí, en partie détruite et restaurée.

3. À la droite de celle-ci, le seul cartel de cette séquence, qui donne brièvement les informations sur la maquette de Gaudí, et uniquement sur elle.

4. À l'extrême droite, un dessin de la fenêtre (sans titre, ni échelle, ni date, ni signature) et une photographie (également sans légende) de sa partie centrale.

Le résultat, pour le visiteur, est une vision kaléidoscopique de cette partie de l'église qu'il a pu, en outre, admirer en vrai sur le chantier. Lui est d'abord proposé un voyage dans le temps, depuis le projet gaudien de cette fenêtre, datant d'un siècle, jusqu'aux dessins de 
Berenguer (1972). Le visiteur se voit également présenter un panorama des différents supports de création des concepteurs de l'église, des maquettes privilégiées par Gaudí jusqu'à la photographie. Ce dernier support, si parlant lorsqu'il s'agit de rendre compte de l'avancement concret du chantier, ne donne ici à voir qu'un ordre de réalité virtuel : une maquette.

Une autre forme de mise en abyme consiste à présenter à différents moments de la visite des « objets» (tout ou partie d'un élément architectural) sous différents supports et qui semblent ainsi se renvoyer les uns aux autres ${ }^{16}$. Ainsi trouve-t-on une vitrine présentant quatre maquettes originales restaurées de ces mêmes "fenêtres des nefs centrales" présentées dans la séquence ci-dessus, également en plâtre ; cette vitrine est quasiment à l'opposé dans le musée (salle $\mathrm{n}^{\circ} 7$ ). Pareille scénographie à la fois redondante et close sur elle-même est cohérente dans le cadre d'un espace cumulant exhibition et production des objets exhibés. Là où les plans de l'œuvre achevée servaient à récapituler la visite dans un but didactique, la mise en abyme démultiplie les représentations possibles d'un même objet, les différents supports, les étapes successives, etc. Loin de servir la même visée pédagogique et simplificatrice, cette autre sorte de contextualisation du chantier « épaissit » la vision du visiteur, le plongeant dans les temporalités multiples de l'œuvre.

\section{Conclusion}

47 Le passé sous le sceau du génie déjà patrimonialisé, la restauration du passé, l'aventure de l'avancement présent du chantier, le mystère et le pari de l'avenir et les coups de pouce du virtuel, sans oublier l'éternité qu'incarnent l'église ${ }^{17}$ et la béatification de son concepteur : le musée n'en finit pas de décliner le chantier de la Sagrada Família à tous les temps.

Le musée se présente comme un lieu d'exhibition "naturelle», assez peu pensé ou organisé. Le discours local assume et revendique cette apparente naïveté muséographique : "Ce qui importe, c'est la construction de l'église », entend-on comme un leitmotiv. Le musée serait alors le simple résultat d'une masse d'archives qu'il a paru intéressant de mettre à la disposition du public. De fait, le musée de la Sagrada Família n'a ni conservateur ni scénographe: les expositions sont le fait des architectes, des maquettistes et des archivistes, et l'inventaire des pièces du musée date seulement de $2003^{18}$. Cette coresponsabilité de la muséographie partagée entre spécialistes d'abord intéressés par l'œuvre elle-même explique sans doute que le musée assume ouvertement la sanctuarisation et le discours hagiographique que la plupart des musées tentent de remettre en cause. L'originalité est ici celle d'un espace entre musée, lieu saint et chantier. Le lien qu'il parvient à établir entre ces différents types d'espaces est garant de l'efficacité du musée, de sa capacité à familiariser les visiteurs avec l'œuvre, à transmettre son histoire et à contextualiser le chantier.

Il ne faut donc pas s'arrêter à l'absence de valorisation du musée (de son concept, de ses fonctions) dans le discours de ses responsables. En effet, dans ce musée d'une œuvre en chantier, la mise en ordre muséographique est perçue comme indispensable en tant que complément logique de l'œuvre visitée. Il s'agit de projeter l'œuvre achevée. Dans ce contexte particulier de chantier où le visiteur d'un jour perçoit d'abord le côté inachevé de l'œuvre, son statut d'œuvre non encore vivante, on comprend l'importance stratégique d'un musée exposant la genèse, l'évolution et le projet final. Par le double jeu 
du virtuel et de la convocation d'un passé mythifié, il s'agit de revendiquer pour une œuvre en devenir une existence immédiate, un sens dès aujourd'hui, le jour même où le visiteur est présent.

\section{BIBLIOGRAPHIE}

BONET, Jordi

Le Temple de la Sagrada Família. Barcelone, Editorial Escudo de Oro.

GALINIER, Jacques, MOLINIÉ, Antoinette

1998 « Le crépuscule des lieux. Mort et renaissance du musée d'anthropologie », Gradhiva 24 :

93-102.

GÓMEZ, Josep, Coll, Jordi, MELERo, Juan C., BURRY, Mark C.

1996 La Sagrada Familia. De Gaudí al CAD. Barcelone, Ediciones de la Universitat Politècnica de Catalanuya.

GRIMAUD, Emmanuel

2003 « Le trésor de Toutânkhamon. Objets, guides et visiteurs au musée du Caire », Gradhiva 34 :

15-28.

HEMMET, Christine

2001 " Musées d'ethnographie d'Asie et d'Occident. Le malaise au présent », Ateliers 23, Temporalité et muséographie. Colloque de l'APRAS. Paris, Laboratoire d'ethnologie et de sociologie : 83-94.

LAHUERTA, Juan José

1993 Antoni Gaudí 1852-1926. Arquitectura, ideologia y politica. Madrid, Electa.

LóPEZ, María Paz

2000 « Devoción a Gaudí antes de su beatificación », La Vanguardia. Barcelone, 31 mai.

MARTINELL, Cèsar

1951 Gaudí i la Sagrada Família, comentada per ell mateix. Barcelone, Ediciones Cossetània.

1967 Gaudí, su vida, su teoría, su obra. Barcelone, Col.legi Oficial d'Arquitectes de Catalunya i Balears.

MICHELET, Dominique

2001 «L'imparfait des passés recomposés. Réflexions sur la perception du temps en archéologie et au musée ", Ateliers 23, Temporalité et muséographie. Colloque de l'APRAS. Paris, Laboratoire d'ethnologie et de sociologie : 11-23.

MOLINIÉ, Antoinette 
2001 « Le blanchiment du temps sale dans les musées d'anthropologie », Ateliers 23, Temporalité et muséographie. Colloque de l'APRAS. Paris, Laboratoire d'ethnologie et de sociologie : 95-100.

NAVARRO ARISA, Juan José

2002 Gaudí. El Architecto de Dios. Barcelone, Planeta Singular.

RECHT, Roland

1999 Le Croire et le Voir. L'Art des cathédrales (XII ${ }^{\mathrm{e}}-\mathrm{XV}^{\mathrm{e}}$ siècle). Paris, Gallimard.

RIVIÈRE, Georges Henri, LEROUX-DHUYS, Jean-François

1990 « Musées et collections publiques, muséologie et muséographie », in Jean Poirier, éd., Histoire des mœurs, vol. III. Paris, Gallimard : 185-217.

SAGRADA FAMÍLIA, LA (DVD)

2005. Barcelone, Canal 7 - Barcelone Multimedia.

SEGALEN, Victor

1995 CEuvres complètes. Édition établie et présentée par Henry Bouillier, vol.I. Paris, Robert Laffont/ Bouquins.

SOSTRES, Josep Maria.

1991 « Situación de la obra de Gaudí en relación con su época y trascendencia actual », in Salvador Tarragó, éd., Antoni Gaudí. Barcelone, Ediciones del Serbal : 45-50.

Temple

1992 « Obertura del Museu ampliat del Temple », juillet-août.

Templo

1961 « Exposición permanente de historia del templo », juillet-août.

1975 « El plano de la Sagrada Familia », novembre.

1977 « Las obras de la Sagrada Familia », avril.

1979 «El jardín de la plaza de Gaudí. Una obra complementaria del Temple de la Sagrada Familia », janvier-février.

Unesco

2003 Brèves descriptions des biens inscrits sur la liste du patrimoine mondial. Paris, Centre $\mathrm{du}$ Patrimoine mondial de l'Unesco.

\section{NOTES}

* Cet article est le résultat de plusieurs terrains à Barcelone, dont le dernier date de septembre/ octobre 2005. Je remercie Laia Vinaixa, responsable du département d'archives de la Sagrada Família, pour son aide.

1. Templo, dont le sous-titre est Revista de la Asociación de Devotos a San José y del Templo Expiatorio de la Sagrada Familia, est également appelé El propagador de la Devoción a San José. Il est publié plusieurs fois par an depuis 1866 et a adopté le catalan (sous le nom Temple) depuis les années 1980. 
2. Pour une étude approfondie de Gaudí, se reporter par exemple à Cèsar Martinell (1967), Josep Maria Sostres (1991) et Juan José Lahuerta (1993).

3. Sur les liens entre temporalité et muséographie, lire le volume d'Ateliers (2001) qui a publié les interventions du colloque de l'APRAS (notamment, sur l'atemporalité des «chefs-d'œuvre», Molinié 2001 : 100).

4. Voir par exemple Rivière et Leroux-Dhuys (1990) pour les fondements historiques de cette comparaison, et Galinier et Molinié (1998[医 : notamment 98 et 102) pour un exemple concret contemporain.

5. Il semble en fait que la municipalité participe financièrement au chantier : la revue Templo parle de 500000 pesetas annuelles en 1961 (1961: 9) et évoque également 10000000 de pesetas d'aide cumulée en 1975 de la part de la Direction générale du patrimoine (1975 : 259).

6. Gaudí était bien sûr conscient du fait que le chantier serait loin d'être achevé à sa mort. Visiblement soucieux de son héritage, il s'attacha à organiser le chantier de son vivant de façon à faciliter le travail de ses successeurs, notamment en leur laissant des espaces achevés en guise de repères techniques et esthétiques.

7. Célèbre sentence de Victor Segalen dans son Journal des îles (1995 : 451).

8. Du moins d'après le texte catalan. Le texte anglais du cartel ne comporte pas cette précision et le texte castillan écrit « la table sur laquelle il travaillait et dormait »!

9. Remarquons que l'église elle-même est frappée du masque de Gaudí, que le sculpteur Subirachs a prêté à l'un des apôtres représenté sur la Façade de la Passion. Subirachs explique cet hommage et précise qu'il s'est servi d'une des rares photographies de l'architecte, qui détestait se faire tirer le portrait.

10. Unesco 2003. Notons que patrimonialisation de l'œuvre et sanctification de l'architecte sont également liées à leurs statuts d'emblèmes de l'identité catalane. En effet, l'histoire de la Sagrada Família est très liée à la catalanité, ne serait-ce que par les convictions politiques de Gaudí. Mais même les orientations esthétiques de Gaudí sont systématiquement rattachées par les commentateurs à la nature catalane : l'histoire veut que l'architecture des tours de la Sagrada Família ait été directement inspirée à l'architecte par les montagnes entourant Barcelone, dans la revendication d'un paysage spécifique que la réappropriation esthétique cristallise en enjeu identitaire. L'Unesco préfère souligner la qualité « vraiment universelle [des œuvres de Gaudí] par la diversité des références culturelles qui les ont inspirées" (Unesco 2003). L'un des principaux enjeux d'une étude de l'église sera de comprendre comment le chantier de la Sagrada Família sert, depuis cent vingt ans, le processus dynamique et parfois chaotique de revendication d'une communauté nationale catalane.

11. Je reprends la numérotation officielle telle que la présente le plan du musée fourni par le département d'archives de la Sagrada Família.

12. Parmi les dates retenues figurent les suivantes (les étoiles marquent les dates notées en gras dans la chronologie) : 1866 : fondation de l'association de dévots de Bocabella ; 1882 : première pierre du chantier par Villar ; 1883 : Gaudí reprend le chantier*; 1889 : fin de la crypte ; 1890 : dessin de la première solution d'ensemble; 1892: début de la Façade de la Nativité ; 1909 : construction de l'école*; 1923 : solution définitive des nefs avec maquettes au $1 / 10 ; 1926$ : mort de Gaudí*; 1930 : 4 clochers de la Façade de la Nativité sont achevés ; 1936 : destructions liées à la guerre civile ; 1954 : commencement de la Façade de la Passion ; 1977 : 4 clochers de la Façade de la Passion sont achevés*; 1986-1990 : fondements des nefs.

13. Ce n'est qu'à l'ultime page de ce livre que Jordi Bonet parle du musée, qu'il intitule «musée monographique de l'histoire de l'église", pour conseiller de clore avec celui-ci la visite du Temple.

14. Le débat ressurgit tous les ans depuis l'après-guerre (Martinell 1951: 119-124). Le 9 janvier 1965, de nombreux intellectuels catalans publièrent une lettre ouverte dans La Vanguardia protestant contre la continuation de l'œuvre de Gaudí malgré la perte des plans originaux et 
malgré son inadéquation, pensaient-ils, aux besoins religieux modernes. Douze ans plus tard, s'adressant au ministre de l'Éducation qui lui demandait où en était cette question, un journaliste la compara aux discussions périodiques sur le monstre du Loch Ness (Templo 1977).

15. Plusieurs autres exemples de mise en abyme pourraient être développés. Citons la maquette restaurée de la Façade de la Nativité réalisée par Gaudí pour l'Exposition universelle de 1910 à Paris, qui est doublée par une photographie d'époque de la maquette présentée lors de cette exposition. Remarquons également que le statut des photographies est assez ambivalent, entre simple témoin des étapes du chantier et objet de musée à part entière. Le musée mélange parfois les dates et les supports, comme pour ce cartel attribuant à une photographie récente la date de 1882 - celle, en réalité, de la réalisation du plan originel de l'église ici photographié.

16. Comme Emmanuel Grimaud l'a montré des objets du « Trésor de Toutankhamon » exposés au musée du Caire (2003).

17. Une des sentences de Gaudí préférées par ses biographes était: "Mon client n'est pas pressé. »

18. L'inventaire récapitule quelque 900 objets, classés suivant la salle du musée correspondante (indice d'une muséographie non évolutive), avec titre, matériau, description, date, échelle et dimensions.

\section{RÉSUMÉS}

L'église catholique de la Sagrada Família, à Barcelone, est un lieu saint monumental dont la construction, entreprise à la fin du XIX ${ }^{\mathrm{e}}$ siècle par l'architecte catalan Antoni Gaudí, est encore de nos jours en pleine activité. Ce chantier comprend un musée qui illustre et concentre la plupart des enjeux de ce lieu. D'une part, le musée ne se contente pas d'exhiber, de façon plus ou moins pédagogique, les étapes et les techniques du chantier; il contribue aussi pleinement à la patrimonialisation de ce lieu saint à venir qu'est la Sagrada Família et à la béatification en cours de son architecte principal. D'autre part, non content de récapituler la visite du chantier que touristes et fidèles effectuent, le musée illustre, à travers des procédés muséographiques souvent originaux, les différentes temporalités du chantier et remplit une fonction de projection de l'œuvre achevée.

The Catholic Sagrada Família church in Barcelona is a monumental holy place whose construction began in the late 19th century under the Catalan architect Antoni Gaudí, and is still underway. The site includes a museum illustrating the history of the place in concentrated form. It is not, however, simply content to educate us with exhibits recounting the various stages and techniques of construction - on the contrary, it contributes fully to patrimonialisation of this holy place in the making and to the ongoing beatification of its main architect. Nor is it content to offer us a simple recapitulation of the mandatory visit to the site by tourists and the faithful, but provides illustration of the site's various temporalities, using some often very original museographic procedures, and gives its visitors an idea of what the finished work will be like. 
INDEX

Mots-clés : construction, Gaudí, lieu saint, musée, temporalité

Keywords : museum, sacred place, temporality

\section{AUTEUR}

MATHIEU CLAVEYROLAS

mathieu.claveyrolas@laposte.net, EHESS, Centre d'études de l'Inde et de l'Asie du Sud (CEIAS) 